\title{
(6) OPEN ACCESS \\ Prevalence of adult Huntington's disease in the UK based on diagnoses recorded in general practice records
}

\author{
Stephen JW Evans, ${ }^{1}$ Ian Douglas, ${ }^{1}$ Michael D Rawlins, ${ }^{1}$ Nancy S Wexler, ${ }^{2,3}$ \\ Sarah J Tabrizi, ${ }^{4}$ Liam Smeeth ${ }^{1}$
}

${ }^{1}$ Department of Epidemiology, London School of Hygiene and Tropical Medicine, University of London, London, UK

${ }^{2}$ Department of Neurology and Psychiatry, Columbia University, New York, New York, USA ${ }^{3}$ Hereditary Disease Foundation, New York, New York, USA

${ }^{4}$ University College London, Institute of Neurology, London,

\section{Correspondence to} Professor Michael D Rawlins, Faculty of Epidemiology and Population Health, London School of Hygiene and Tropical Medicine, Keppel Street, London WC1E 7HT, UK; michael.rawlins@nice.org.uk

Received 30 November 2012 Revised 17 February 2013 Accepted 20 February 2013 Published Online First 12 March 2013

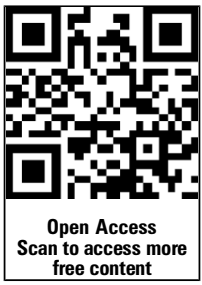

To cite: Evans SJW, Douglas I, Rawlins MD, et al. I Neurol Neurosurg Psychiatry 2013;84: 1156-1160.

\section{ABSTRACT}

Background and purpose The prevalence of Huntington's disease (HD) in the UK is uncertain. Recently, it has been suggested that the prevalence may be substantially greater than previously reported. This study was undertaken to estimate the overall UK prevalence in adults diagnosed with $\mathrm{HD}$, using data from primary care.

Methods The electronic medical records of patients aged 21 years or more, with recorded diagnoses of HD, were retrieved from the UK's General Practice Research Database. Prevalence was estimated from the number of persons with recorded diagnoses of $\mathrm{HD}$, on 1 July each year, between 1990 and 2010. This number was divided by the total number of persons registered with participating general practices on that same date. These data were also used to estimate both age specific prevalence and prevalence in various regions of the UK.

Results A total of 1136 patients diagnosed with HD, aged 21 years or more, were identified from the database. The estimated prevalence (expressed per 100000 population) rose from $5.4(95 \% \mathrm{Cl} 3.8$ to 7.5$)$ in 1990 to $12.3(95 \% \mathrm{Cl} 11.2$ to 13.5$)$ in 2010. Although an increased prevalence was observed within every age group, the most dramatic was in older patients. Age specific prevalence was highest in the $51-60$ year age range $(15.895 \% \mathrm{Cl} 9.0$ to 22.3$)$. The prevalence of adult HD was lowest in the London region $(5.4(95 \% \mathrm{Cl} 3.0$ to 8.9$))$ and highest in the North East of England (18.3 (95\% Cl 8.6 to 34.6)) and Scotland (16.1 (95\% Cl 10.8 to 22.9$)$ ).

Conclusions The prevalence of diagnosed HD is clearly substantially higher in the UK than suggested from previous studies. By extrapolation to the UK as a whole, it is estimated that there are more than 5700 people, aged 21 years or more, with HD. There has also been a surprising doubling of the HD population between 1990 and 2010. Many factors may have caused this increase, including more accurate diagnoses, better and more available therapies and an improved life expectancy, even with HD. There also appears to be a greater willingness to register a diagnosis of HD in patients' electronic medical records. Such a high prevalence of HD requires more ingenuity and responsiveness in its care. How to appropriately care for, and respond to, so many individuals and families coping with the exigencies of HD demands our greatest resolve and imagination.

\section{INTRODUCTION}

Huntington's disease (HD) is a progressive, fatal neurodegenerative disorder causing abnormal movements, psychiatric disturbances and cognitive decline. ${ }^{1}{ }^{2} \mathrm{HD}$ segregates as an autosomal dominant trait located on chromosome $4 \mathrm{p} 16.3 .^{3}$ The HD abnormality is due to an expansion of a polyglutamine (CAG) repeat in exon 1 of the huntingtin protein. $^{2}$ The size of the CAG repeat is the most critical determinant for the age of onset of HD symptoms. ${ }^{2}$ Alleles with fewer than 34 CAGs rarely produce symptoms; alleles between 35 and 39 CAGs have variable penetrance; alleles of 40 CAGs or more are fully penetrant if a person lives a normal lifespan; and alleles of 60 CAGs or greater usually result in juvenile onset under 21 years of age. Including all repeat lengths, the size of the CAG repeat accounts for $72 \%$ of the variability in age of onset. ${ }^{2}$ However, $90 \%$ of all people with HD worldwide have between 40 and 50 CAGs. For these individuals, the repeat length accounts for only $40 \%$ of the variability in age of onset. Of this remaining variability in the age of onset, genetic factors, other than the HD gene, account for $60 \%$ and environmental factors for $40 \% .^{2}$ Genetic and environmental modifiers could be playing a role by influencing the prevalence of $\mathrm{HD}$ in the UK and globally.

Over the past 60 years there have been many attempts to estimate the prevalence of $\mathrm{HD}$ in the UK. Most of these studies attempted to enumerate the numbers of patients by searching general practice, hospital or nursing home records, mainly in specific geographical regions. ${ }^{4-6}$ Overall, these investigations suggested a prevalence of $\mathrm{HD}$ of approximately 6 per 100000 . These estimates have been questioned ${ }^{7}$ recently because of the apparent disparity between these estimates and the numbers of patients receiving services from the Huntington's Disease Association.

A reliable estimate of the overall prevalence of $\mathrm{HD}$, as well as of regional differences, is important for planning and delivering the appropriate provision of health and social care. The present study was undertaken to obtain a contemporary estimate of the prevalence of diagnosed HD in the UK as a whole, as well as to explore the extent of any regional differences. The incidence and prevalence of the juvenile form of HD is the subject of a separate study. ${ }^{8}$ 


\section{METHODS}

Study design and setting

The General Practice Research Database (GPRD) is the world's largest computerised database of anonymised longitudinal medical records from primary care. It is now included under the umbrella of the Clinical Practice Research Datalink which brings together data from across the UK's National Health Service. The GPRD is assembled from the electronic health records of patients registered with contributing general practices. Currently over 5 million patients from approximately 625 contributing practices are included. The practices are broadly representative of those in the UK in geographical distribution, practice size, and the age and sex of registered patients. Each individual patient is assigned a unique identification number. No information from medical records allowing identification of patients is included in the database. The data are entirely anonymous to investigators.

The GPRD aims to include complete diagnostic and prescribing information for each registered patient. When patients newly register with a contributing practice, major past and existing diseases are recorded in their medical records and are included in the research database. However, the dates of onset and dates of past diagnoses are not always accurately recorded. Some diagnoses that occurred in the past may be recorded without a date or as occurring at the date of registration. Morbidity in UK primary care is recorded using Read codes Clinical Terms V.3. ${ }^{9}$ At both practice and individual patient levels the data are subject to a range of quality checks prior to being made available for research purposes. ${ }^{10}$ The quality of the data has been found to be high in a large number of independent validation studies. ${ }^{11}$

Ethics approval for the study was obtained from the GPRD's Independent Scientific Advisory Committee and from the London School of Hygiene and Tropical Medicine's Research Ethics Committee. The potential funders of the study played no part in its design, analysis or interpretation.

\section{Participants and variables}

The source population is all patients aged 21 years or more who registered with general practices contributing to the GPRD during the period 1990-2010. Eligible cases are defined as persons with one or more recorded diagnoses of HD or Huntington's chorea anywhere in their medical records. The Read codes used to identify cases of HD were F134.00 (Huntington's chorea) and Eu02200 (dementia in HD).

For each general practice, the observation period for the study began as the later of two dates: either the study start date, 1 January 1990, or the date at which the practice started contributing research standard data to the GPRD. The end of the observation period was the earlier of two dates: the last date for which the practice contributed data to the GPRD or the study end date, 31 December 2010. Individual patients were included in denominators only during times within the observation period that they were registered with a practice contributing data to the GPRD.

\section{Bias}

The records of patients diagnosed with rheumatic and Sydenham's chorea (Read codes G02.00, GO2.11, GO20.00, GO21.00 and GO2z.00), 'drug induced chorea' (135 200) and 'other choreas' (F135z00 and F135.00) were reviewed to assess the extent to which recorded diagnoses of other forms of chorea might represent possible cases of HD. An approximate
$20 \%$ random sample of the full records of cases with 'other choreas' was examined in detail. They were categorised independently as 'not HD', 'possibly HD', 'probably HD' and 'definite HD' by the authors (NSW, SJT and MDR).

\section{Statistical methods}

Prevalence was estimated from the ratio of number of persons with a recorded diagnosis of HD on 1 July each year, between 1990 and 2010, divided by the total number of persons in the database, on that same date. Binomial CIs were calculated. In estimating the prevalence in age bands, and among various geographical regions of the UK, annual point prevalence estimates were averaged and approximate (binomial) 95\% CIs calculated. All prevalence rates are expressed per 100000 of the population.

\section{RESULTS}

\section{Participants}

A total of 1136 patients aged 21 years or more with a diagnosis of HD were identified in the database. Of those with recorded diagnoses, 592 were women and 544 were men.

\section{Main findings}

Between 1990 and 2010, the average prevalence of HD was 10.0 (95\% CI 8.8 to 11.3 ). Prevalence was similar in women $(10.4,95 \%$ CI 8.7 to 12.3$)$ and men $(9.4,95 \%$ CI 7.8 to 11.4$)$. However, the prevalence of HD (table 1) more than doubled over the two decades, from 5.4 (95\% CI 3.8 to 7.5 ) in 1990 to 12.3 (11.2 to 13.5 ) in 2010. All age groups had an increase in prevalence. This was most pronounced among older patients (table 2). The average prevalence rates of HD in different age bands, across the whole period, are shown in table 3. As

Table 1 Point prevalence rates of Huntington's disease by calendar year (per 100 000)

\begin{tabular}{|c|c|c|c|}
\hline Year & $\begin{array}{l}\text { Population } \\
\text { numbers }\end{array}$ & $\begin{array}{l}\text { No of cases } \\
\text { with recorded } \\
\text { diagnoses of HD }\end{array}$ & $\begin{array}{l}\text { Prevalence of HD } \\
(95 \% \mathrm{Cls})\end{array}$ \\
\hline 1990 & 663493 & 36 & 5.4 (3.8 to 7.5 ) \\
\hline 1991 & 899206 & 57 & 6.3 (4.8 to 8.2 ) \\
\hline 1992 & 1012540 & 65 & 6.4 (4.6 to 8.2 ) \\
\hline 1993 & 1155285 & 74 & 6.4 (5.0 to 8.0 ) \\
\hline 1994 & 1250401 & 94 & 7.5 (6.1 to 9.2 ) \\
\hline 1995 & 1334767 & 96 & 7.2 (5.8 to 8.8 ) \\
\hline 1996 & 1507407 & 107 & 7.1 (5.8 to 8.6 ) \\
\hline 1997 & 1800025 & 143 & 7.9 (6.7 to 9.4$)$ \\
\hline 1998 & 2003819 & 162 & 8.1 (6.9 to 9.4$)$ \\
\hline 1999 & 2358898 & 199 & 8.4 (7.3 to 9.7$)$ \\
\hline 2000 & 2812169 & 238 & 8.5 (7.4 to 9.6$)$ \\
\hline 2001 & 3065401 & 285 & 9.3 (8.3 to 10.4$)$ \\
\hline 2002 & 3308580 & 317 & 9.6 (8.6 to 10.7$)$ \\
\hline 2003 & 3397539 & 335 & 9.9 (8.8 to 11.0$)$ \\
\hline 2004 & 3510661 & 386 & 11.0 (9.9 to 12.2$)$ \\
\hline 2005 & 3599761 & 405 & $11.3(10.2$ to 12.4$)$ \\
\hline 2006 & 3603973 & 412 & 11.4 (10.4 to 12.6$)$ \\
\hline 2007 & 3628381 & 409 & 11.3 (10.2 to 12.4$)$ \\
\hline 2008 & 3610472 & 439 & $12.1(11.1$ to 13.4$)$ \\
\hline 2009 & 3591467 & 446 & $12.4(11.3$ to 13.6$)$ \\
\hline 2010 & 3515986 & 432 & $12.3(11.2$ to 13.5$)$ \\
\hline
\end{tabular}


Table 2 Average prevalence rates of Huntington's disease, 1990-2010, by 7 year periods (per 100000 population)

\begin{tabular}{llll}
\hline & \multicolumn{4}{l}{ Averages and 95\% Cls } \\
\cline { 2 - 4 } Age (years) & $1990-1996$ & $1997-2003$ & 2004-2010 \\
\hline $21-39$ & $5.1(3.8$ to 6.7$)$ & $5.6(4.5$ to 6.5$)$ & $6.0(5.1$ to 7.1$)$ \\
$40-49$ & $12.0(9.1$ to 15.5$)$ & $14.1(11.0$ to 16.5$)$ & $17.9(15.8$ to 20.3$)$ \\
$50-59$ & $16.5(17.8$ to 21.1$)$ & $18.5(15.9$ to 21.4$)$ & $22.0(19.4$ to 24.9$)$ \\
$60-69$ & $12.6(9.1$ to 17.1$)$ & $18.5(15.5$ to 21.9$)$ & $24.2(21.1$ to 27.5$)$ \\
$70+$ & $7.2(4.8$ to 10.4$)$ & $10.8(8.7$ to 13.3$)$ & $15.6(13.2$ to 18.3$)$ \\
\hline
\end{tabular}

expected, prevalence was low in younger age groups. It rises to a peak between the ages of 51 and 65 years.

The regional prevalence rates of recorded diagnoses of $\mathrm{HD}$ showed substantial heterogeneity (table 4). The highest rates were in Scotland and North East England. The lowest rates were in London.

\section{Other choreiform diagnoses}

In addition to people with a recorded diagnosis of HD, 866 patients had a recorded diagnosis of rheumatic or Sydenham's chorea, 566 had a diagnosis of other chorea and 12 had a diagnosis of drug induced chorea. The median year of birth of patients with recorded diagnoses of rheumatic or Sydenham's chorea was 1941. Of these, 148 (17\%) were born after 1960. In only 31 instances were diagnoses of rheumatic or Sydenham's chorea subsequently revised to HD and all these are included in the estimates for HD reported here. From the detailed examination of the records of a sample $(n=115)$ of 'other choreas', the adjudication indicated that 101 patients had no evidence to support a diagnosis of HD and 14 were possible cases. Of the people with medical codes for chorea in whom we found no evidence of HD, 52 had a diagnosis of 'other chorea' in childhood or adolescence yet were all alive and well decades later. We are confident these records represent rheumatic chorea in childhood or adolescence. The 49 remaining people with medical codes for chorea had other likely underlying causes such as cerebrovascular disease or thyrotoxicosis. In four

Table 3 Average prevalence rates, 1990-2010, of recorded diagnoses of Huntington's disease by age group (per 100000 )

\begin{tabular}{llll}
\hline $\begin{array}{l}\text { Age group } \\
\text { (years) }\end{array}$ & $\begin{array}{l}\text { Average population } \\
\text { numbers }\end{array}$ & $\begin{array}{l}\text { Average HD } \\
\text { numbers }\end{array}$ & $\begin{array}{l}\text { Average prevalence } \\
\text { (95\% Cls) }\end{array}$ \\
\hline $21-25$ & 202852 & 4 & $2.0(0.5$ to 5.1$)$ \\
$26-30$ & 223488 & 7 & $3.1(1.3$ to 6.5$)$ \\
$31-35$ & 240698 & 15 & $6.2(3.5$ to 10.3$)$ \\
$36-40$ & 249476 & 23 & $9.2(5.8$ to 13.8$)$ \\
$41-45$ & 241745 & 28 & $11.6(7.7$ to 16.7$)$ \\
$46-50$ & 225037 & 32 & $14.2(9.7$ to 20.1$)$ \\
$51-55$ & 208745 & 33 & $15.81(10.9$ to 22.2$)$ \\
$56-60$ & 193437 & 30 & $15.8(10.5$ to 22.1$)$ \\
$61-65$ & 169441 & 26 & $15.3(10.0$ to 22.5$)$ \\
$66-70$ & 144295 & 21 & $14.6(9.0$ to 22.3$)$ \\
$71-75$ & 125812 & 13 & $10.3(5.5$ to 17.7$)$ \\
$76-80$ & 102360 & 9 & $8.8(4.0$ to 16.7$)$ \\
$81-85$ & 72092 & 4 & $5.6(1.5$ to 14.2$)$ \\
$86+$ & 59105 & 1 & $1.7(0$ to 9.4$)$ \\
\hline
\end{tabular}

$H D$, Huntington's disease.
Table 4 Average prevalence rates of Huntington's disease, for 1990 to 2010 , by UK region (per 100000 )

\begin{tabular}{lccc}
\hline Region & $\begin{array}{l}\text { Average } \\
\text { population } \\
\text { numbers }\end{array}$ & $\begin{array}{l}\text { Average } \\
\text { HD numbers }\end{array}$ & $\begin{array}{l}\text { Average } \\
\text { prevalence } \\
\text { (95\% Cls) }\end{array}$ \\
\hline England & 198285 & 19 & $9.6(5.8$ to 15.0$)$ \\
North East & 49317 & 31 & $18.3(8.4$ to 34.6$)$ \\
North West & 310454 & 31 & $10.0(6.8$ to 14.2$)$ \\
Yorkshire and Humber & 116186 & 13 & $11.2(6.0$ to 19.1$)$ \\
Midlands & 163149 & 15 & $9.2(5.2$ to 15.2$)$ \\
East of England & 248603 & 24 & $9.7(6.2$ to 14.4$)$ \\
South West & 191191 & 18 & $9.4(5.6$ to 14.9$)$ \\
South Central & 259279 & 26 & $10.0(6.6$ to 14.7$)$ \\
London & 278859 & 15 & $5.4(3.0$ to 8.8$)$ \\
South East & 202667 & 22 & $10.9(6.8$ to 16.4$)$ \\
Northern Ireland & 79397 & 6 & $7.6(2.8$ to 16.5$)$ \\
Scotland & 186902 & 6 & $16.1(10.8$ to 22.9$)$ \\
Wales & 209430 & 18 & $8.6(5.1$ to 13.6$)$ \\
\hline
\end{tabular}

HD, Huntington's disease.

records the diagnosis of 'other chorea' was subsequently revised to some other movement disorder, such as Parkinson's disease. The 14 possible cases of HD had 'other chorea' recorded together with psychiatric symptoms, mainly depression and/or anxiety. These cases might reflect an underlying diagnosis of HD or, alternatively, an HD phenocopy disorder. ${ }^{12}$

\section{DISCUSSION}

In 2010, our estimate of the prevalence of diagnosed adult HD was 12.3 per 100000 people (table 1). Other studies undertaken in the UK between 1970 and 1995 showed an estimated prevalence of HD of approximately 6 per 100000 , with some suggestions of geographical variation. ${ }^{4}$ The most recent published estimate, ${ }^{6}$ based on data from 2004 to 2007, suggested prevalence rates ranging from 6.4 to 6.6 per 100000 . Our substantially larger estimate of the prevalence of HD in the UK is closer to that recently suggested based on the numbers of people receiving services from the voluntary sector. ${ }^{7}$

The prevalence estimates reported here relate to diagnosed $\mathrm{HD}$ as recorded in primary care health records. Given the rarity of the disorder, and the expertise needed to diagnose it, specialists would have made the recorded diagnoses. The GPRD records do not include reports of individual patient's CAG repeat lengths. However, we are confident that genetic tests will have been consistently used by UK specialists since the mid-1990s in order to diagnose HD. Consequently, we do not consider that our results will have been confounded by the inclusion of patients with HD phenocopy syndromes. ${ }^{12}$ It is therefore extremely unlikely that our results overestimate the true prevalence. We accept that we might have failed to capture all cases and our current prevalence figure probably still represents an underestimate.

Our analysis of the data derived from GPRD documents a dramatic and significant increase in the prevalence of HD over the past two decades (table 1). Many explanations can account for this increase. Since the mid-1990s, genetic testing has been routinely used in the UK to definitively confirm the presence of the expanded allele in the HD gene. Patients presenting with atypical manifestations of $\mathrm{HD}$ are now being correctly diagnosed. These include patients with so-called 'senile chorea'. Very elderly patients, even those presenting in their 80 s or 90 s, 
are now being diagnosed with HD. In addition, patients with no family history, and those with new mutations, are being correctly diagnosed using the HD genetic test.

Our data are also compatible with a genuine rise in prevalence. ${ }^{13}$ Many factors influence patient longevity. Effective symptomatic treatments can help extend life. Tetrabenazine can reduce chorea and minimise the risks of falls and choking. Antidepressants and antipsychotic treatments may reduce suicide rates which are 7-12 times more common in $\mathrm{HD}{ }^{2}$ Attention to good nutrition is also critical because patients need to consume as much as 5000 calories daily to maintain their weight. Pureed foods and other forms of nutritional support, swallowing therapy and enterogastrostomy feeding can add years to peoples' lives. Measures that increase the longevity of the population as a whole, such as those reducing the risk of cardiovascular disease, may also contribute to an increase in life expectancy of those with HD.

HD has been heavily stigmatised globally for over a century. We know that some UK patients request that their hospital specialists refrain from informing their general practitioners of their HD diagnosis. Some diagnoses of HD made by specialists, and communicated to general practitioners, may not be recorded in their records due to fears with respect to issues of confidentiality. A confirmed diagnosis of HD in one family member has genetic consequences for the rest of the family, who may not realise that they are at risk. Individuals diagnosed with HD may lose the capacity to obtain life insurance or a mortgage. Many unanticipated and adverse consequences for patients and families may appear, just from a recorded diagnosis of HD. However, over the two decades of our study, there may have been less reluctance to conceal a diagnosis of HD. This may lead to a more accurate reporting of the true prevalence over time. At every age group, there was an increased recording of HD diagnoses. However, the increase was most marked in the later age groups.

In all respects, our data are internally valid. The proportion of affected men and women were similar. Prevalence was highest in patients aged 51-65 years. There was no evidence to suggest that individuals with diagnoses of rheumatic or Sydenham's chorea had underlying HD. It is possible that a small number of the 14 patients, out of the 121 records subjected to close scrutiny with recorded diagnoses of 'other choreas' and categorised by us as 'possible' cases, actually had HD. If we include these individuals, our prevalence estimates increase by approximately $6 \%$.

We noted marked regional variation in prevalence across the UK (table 4). This may partly be due to random error but may also reflect genuine regional differences. Previous studies ${ }^{4}$ have suggested higher prevalence rates in Scotland. No studies have been reported from North East England. The lower rates in London may be the result of the net effects of migration.

The recent study by Sackley and colleagues ${ }^{6}$ of the prevalence of HD in the UK as a whole was based on an analysis of the THIN (The Health Improvement Network) database. ${ }^{14}$ Their estimate, for the years 2004 to 2007 , ranged from 6.0 to 6.5 per 100000 . It is unclear why these prevalence estimates are so much lower than the ones reported using GPRD. While differences between the data sources (such as quality checks and the specification of periods of follow-up from contributing practices) may have made a small contribution to the differences in prevalence in our study, compared with that of Sackley and colleagues, ${ }^{6}$ it is likely that issues of methodological design explain most of the discrepancy. Sackley and colleagues ${ }^{6}$ adjusted their prevalence estimates of HD by including all patients with a diagnosis of 'chorea' as well as half of those with a family history of HD. Using this approach, their revised 'total' prevalence estimates, between 2004 and 2007, ranged from 9.6 to 10.3 per 100000 , which is still less than the prevalence reported in the present study. Although it is possible that some patients with a diagnosis of 'chorea' did indeed have HD, Sackley et al's revised estimate neglects the fact that 'chorea' is a symptom of many other conditions. From our data it would have been inappropriate to have made any adjustments to our estimates of prevalence by including other diagnoses of chorea.

Sackley and colleagues ${ }^{6}$ included half of those people who had a recorded family history of HD in their revised number of cases. However, they do not specify if a family history of HD means that the person had an affected parent or grandparent. They assumed that half of those at risk will develop symptoms of HD, which is not true. They also make the egregious mistake of including these at risk individuals as already diagnosed in their yearly prevalence calculations of diagnosed HD patients. When estimating the prevalence of patients with diagnosed HD, it is inappropriate to include individuals who are merely at risk. Nor should prevalence estimates include people whose genetic testing reveals an expanded CAG repeat. Having an expanded CAG repeat is not synonymous with a clinical diagnosis of HD.

There are 46638400 people over the age of 21 years in the UK population. ${ }^{15}$ Applying our current prevalence estimate of 12.3 per 100000 argues for more than 5700 people in the UK with diagnosed HD. A much larger population of people ${ }^{16}$ car- $^{-}$ rying expanded CAG repeats are destined to follow the relentless, irrevocable and destructive attack on body and mind that HD presents. The misery and suffering HD brings to patients and families and the growing scale of the problem behoves us to provide the best health and social care. It is also important to replace our current trial and error use of symptomatic treatments with therapies that are fit for purpose. We must develop new symptomatic therapies aimed at the myriad motor, cognitive and psychiatric problems that HD represents. And even more urgent is our task to create innovative treatments and cures aimed at stopping the abnormal protein through gene silencing or repairing the expanded repeat. The fact that the HD mutation is the same worldwide gives us hope that alleviation of suffering with new therapies and cures will lift a global burden.

Correction notice This paper has been amended since it was published Online First. The authors have noticed an error in the last sentence of the penultimate paragraph. The original version stated: "Having an expanded CAG repeat is synonymous with a clinical diagnosis of HD." It has now been corrected to read "Having an expanded CAG repeat is not synonymous with a clinical diagnosis of HD."

Acknowledgements The authors are grateful to the Hereditary Disease Foundation and the Huntington's Disease Association for their support for this study.

Contributors SJWE, MDR and LS originally conceived the study. SJWE and ID extracted the data from the General Practice Research Database and undertook the statistical analyses. MDR, SJT and NSW examined the individual patient records. All authors contributed to the interpretation of the results and the writing of the paper. MDR is the guarantor.

Funding This work was supported by the Huntington's Disease Association and Hereditary Disease Foundation. LS is supported by a Senior Clinical Fellowship from the Wellcome Trust and ID by a fellowship from the Medical Research Council.

Competing interests None.

Ethics approval The study was approved by the General Practice Research Database's Independent Scientific Advisory Committee and the London School of Hygiene and Tropical Medicine's Research Ethics Committee.

Provenance and peer review Not commissioned; externally peer reviewed. 
Open Access This is an Open Access article distributed in accordance with the Creative Commons Attribution Non Commercial (CC BY-NC 3.0) license, which permits others to distribute, remix, adapt, build upon this work non-commercially, and license their derivative works on different terms, provided the original work is properly cited and the use is non-commercial. See: http://creativecommons.org/licenses/by-nc/3.0/

\section{REFERENCES}

1 Novak MJ, Tabrizi SJ. Huntington's disease. BMJ 2010;341:34-40.

2 Wexler NS. Huntington's disease: advocacy driving science. Annu Rev Med 2012;63:1-22.

3 Gusella JF, Wexler NS, Conneally PM, et al. A polymorphic DNA marker genetically linked to Huntington's disease. Nature 1983;306:234-8.

4 Harper PS. The epidemiology of Huntington's disease. In: Bates G, Harper PS, Jones L, eds. Huntington's disease. 3rd edn. Oxford: Oxford University Press, 2002:159-97.

5 Hoppitt T, Pall H, Calvert M, et al. A systematic review of the incidence and prevalence of long-term neurological conditions in the UK. Neuroepidemiology 2011;36:19-28.

6 Sackley C, Hoppitt TJ, Calvert M, et al. Huntington's disease: current epidemiological and pharmacological management in UK primary care. Neuroepidemiology 2011;37:216-21.

7 Rawlins M. Huntington's disease out of the closet? Lancet 2010;1372-3.
8 Douglas I, Evan S, Rawlins MD, et al. Juvenile Huntington's disease: a populationbased study using the General Practice Research Database. BMJ Open 2013;3(4). pii: e002085. doi: 10.1136/bmjopen-2012-002085.

9 NHS Connecting for Health. Read Codes. http://www.connectingforhealth.nhs.uk/ systemsandservices/data/uktc/readcodes (accessed 20 Aug 2012).

10 Clinical Practice Research Datalink. CPRD Governance. http://www.cprd.com/ governance/ (accessed 20 Aug 2012).

11 Herrett E, Thomas SL, Schoonen WM, et al. Validation and validity of diagnoses in the General Practice Research Database: a systematic review. Br J Clin Pharmacol 2010;69:4-14.

12 Wild EJ, Tabrizi SJ. The differential diagnosis of chorea. Pract Neurol 2007:7:360-73.

13 Morrison PJ. Accurate prevalence and uptake of testing for Huntington's disease. Lancet 2010;9:1147.

14 Bourke A, Dattani H, Robinson M. Feasibility study and methodology to create a quality-evaluated database of primary care data. Inform Prim Care 2004;12: $171-7$.

15 Office of National Statistics. http:www.ons.gov.uk/publications/re-reference-tables. html?edition=tcm\%3A77-23187 (accessed 20 Aug 2012).

16 Conneally PM. Huntington's disease: genetics and epidemiology. Am I Hum Genet 1984;36:506-26. 